\title{
Editorial
}

\section{Fecundidad en Chile: 2000-2008}

La tendencia de la fecundidad de un país es producto de una serie de variables, entre otras, sociales, educacionales, sanitarias, económicas, como también del acceso de la población, especialmente femenina, a los métodos de planificación familiar y de anticoncepción.

Chile se encuentra en el denominado proceso demográfico de transición avanzada, lo que implica una baja tasa de natalidad (2008: 14,8/1000 habitantes) y de mortalidad (5,4/1000 habitantes) que trae como consecuencia un crecimiento natural bajo y el envejecimiento poblacional (1). En 1990, Chile alcanzó la natalidad más alta en su historia demográfica con 309.220 nacidos vivos, posteriormente inicia un continuo descenso, año tras año hasta 2004 , equivalente a $21,2 \%$ (2), para posteriormente iniciar un discreto aumento.

El control de la fecundidad es una de las principales estrategias de salud pública, para el control de la morbimortalidad materna, perinatal e infantil, especialmente en los grupos de mayor riesgo reproductivo (3). En la actualidad, el libre acceso a la anticoncepción hace que la tasa global de fecundidad del país sea de 1,92/hijos-mujer, inferior a la tasa de recambio poblacional (2,1/hijos-mujer), de ahí que es necesario el desarrollo de políticas públicas que incentiven la natalidad.

No se ha establecido cual es la edad de las mujeres donde ellas enfrenten el menor riesgo reproductivo, sin embargo, es reconocido el mayor riesgo en las edades extremas de la edad fértil, de ahí que los estudios de riesgo reproductivo establezcan habitualmente como grupo control a las mujeres entre los 20 y 34 años. Además de la edad materna, otro importante factor de riesgo son las enfermedades maternas pregestacionales, que también están fuertemente asociadas a las mujeres en edad materna avanzada ( $\geq 40$ años).

Todo lo anterior indica que se debe estimular la natalidad en mujeres de menor riesgo reproductivo y ser muy cautos frente a los grupos de riesgos. Esto no significa una discriminación hacia los gru- pos de riesgo, sino que especialmente ellas, deben planificar su embarazo, estando informadas de su condición de mayor riesgo materno-perinatal, para decidir el mejor momento para enfrentar una gestación con todo el apoyo del equipo de salud, para que sean madres, pero con un riesgo controlado.

Los cambios culturales de la población hacen que el inicio de la vida coital sea más precoz, con el consiguiente aumento de los nacimientos en las adolescentes, con todas las consecuencias biológicas y sociales que conlleva. A su vez la mayor oportunidad y/o necesidad laboral de las mujeres, hace que estas posterguen la maternidad a edades mayores, siendo Chile un país de fecundidad de tipo tardío por presentar una edad promedio al momento del primer hijo de 28 años y concentrarse la fecundidad mayoritariamente en el grupo etario de 25-29 años (4). Esto último, más el envejecimiento poblacional de la mujeres en edad fértil, hace que los nacimientos en mujeres de 40 o más años haya aumentado significativamente.

Estos antecedentes hace necesario que los obstetras-ginecólogos, matronas y enfermerasmatronas, responsables del cuidado de la salud materna-infantil, estén informados de la evolución de los cambios demográficos para planificar junto a las autoridades de salud, las estrategias que permitan controlar y prevenir las situaciones de mayor riesgo reproductivo que enfrenta el país, de ahí que se estime necesario tener un permanente conocimiento de los cambios demográficos del país, que se presentan muy resumidamente lo que sucede en Chile en los primeros 9 años del siglo XXI (Tabla I).

En el periodo 2000-2008 hubo un total de 2.148.033 nacidos vivos e inscritos, con una tendencia descendente no significativa $(r:-0,281 ; p=0,464)$. Sin embargo, a partir de 2005 se aprecia un aumento de los nacidos vivos equivalente a un 6,9\% acumulado. Según el rango de edad materna, solo las mujeres de 40-44 y 45-49 años presentaron en el período una tendencia de la natalidad significativamente ascendente. Otro antecedente importante 
Tabla I

TENDENCIA DE LA FECUNDIDAD EN CHILE: 2000-2008

\begin{tabular}{ccccccccccc}
\hline \multicolumn{10}{c}{ Nacidos vivos según rango de edad materna (años) } \\
Año & $<15$ & $15-19$ & $20-24$ & $25-29$ & $30-34$ & $35-39$ & $40-44$ & $45-49$ & $\geq 50$ & Total \\
\hline 2000 & 1.055 & 39.257 & 57.141 & 63.812 & 50.675 & 29.471 & 7.119 & 359 & 4 & 248.893 \\
2001 & 1.162 & 38.722 & 55.892 & 61.735 & 50.651 & 30.146 & 7.420 & 371 & 17 & 246.116 \\
2002 & 1.118 & 36.500 & 55.414 & 58.775 & 49.575 & 29.450 & 7.821 & 326 & 2 & 238.981 \\
2003 & 994 & 33.838 & 54.536 & 56.443 & 50.557 & 29.662 & 8.087 & 362 & 7 & 234.486 \\
2004 & 905 & 33.507 & 53.512 & 54.126 & 50.571 & 29.108 & 8.207 & 333 & 2 & 230.271 \\
2005 & 935 & 35.143 & 54.032 & 53.401 & 49.906 & 28.810 & 8.234 & 364 & 6 & 230.831 \\
2006 & 954 & 36.819 & 54.312 & 52.387 & 49.335 & 28.767 & 8.372 & 430 & 7 & 231.383 \\
2007 & 955 & 38.650 & 56.174 & 56.429 & 50.151 & 29.438 & 8.324 & 394 & 9 & 240.524 \\
2008 & 1.025 & 39.902 & 57.915 & 58.338 & 50.391 & 30.234 & 8.307 & 436 & 0 & 246.548 \\
Total & 9.103 & 332.338 & 498.928 & 515.446 & 451.812 & 265.086 & 71.891 & 3375 & 54 & 2.148 .033 \\
$\%$ & 0,42 & 15,47 & 23,23 & 24,00 & 21,03 & 12,34 & 3,35 & 0,16 & 0,003 & 100,0 \\
$r$ & $-0,594$ & 0,083 & 0,038 & $-0,642$ & $-0,348$ & $-0,114$ & 0,893 & 0,697 & $-0,280$ & $-0,281$ \\
Valor p & 0,092 & 0,832 & 0,923 & 0,062 & 0,359 & 0,770 & 0,001 & 0,037 & 0,465 & 0,464 \\
\hline
\end{tabular}

es que los recién nacidos vivos de las madres $<20$ años y las $\geq 35$ años constituyen el $31,7 \%$ del total de los nacimientos, grupos etarios de reconocido alto riesgo materno perinatal. Las adolescentes por mayor riesgo de mortalidad neonatal e infantil, por bajo peso al nacer y parto prematuro (5), mientras que las mujeres de 35 o más años por mayor riesgo de mortalidad perinatal, infantil y materna $(6,7,8)$.

Es importante destacar que las adolescentes desde 2005, luego de una franca tendencia al descenso de la natalidad presentan una preocupante tendencia al ascenso, lo que sumado a la tendencia ascendente de los nacimientos en mujeres de 400 más años hace que los indicadores de riesgo de mortalidad materna, perinatal e infantil presenten un estancamiento en los últimos años (1).

El embarazo adolescente es la principal causa de abandono escolar y de la perpetuación de la pobreza, por lo que es esperanzadora la incorporación por el Ministerio de Educación, de siete programas de educación sexual y afectividad a partir de la educación básica (9), sin embargo, es necesario fortalecer los programas de control de la fecundidad en la adolescencia, especialmente por el repunte del embarazo adolescente que se aprecia desde el 2005.

En conclusión: en el período 2000-2008 hay una natalidad estable, con discreta tendencia al ascenso desde 2005; hay una preocupante tendencia al ascenso del embarazo adolescente; hay un significativo ascenso de la natalidad en mujeres de 40 o más años y casi un tercio de los recién nacidos pertenecen a mujeres etariamente de mayor riesgo. Todo lo anteriormente indica que Chile tiene una población obstétrica de creciente riesgo materno, perinatal e infantil, y que los profesionales encargados del cuidado de la salud de la madre y del niño enfrentan un importante desafío.

Dr. Enrique Donoso.

Editor Revista Chilena de Obstetricia y Ginecología.

\section{REFERENCIAS}

1. Instituto Nacional de Estadísticas. Estadísticas Vitales. Informe Anual 2008.

2. Donoso E, Carvajal J, Domínguez MA. [Fecundity reduction and aging in fertile women population in the period 1990-2004 in Chile] Rev Med Chil 2009;137:766-73.

3. Stover J, Ross J. How increased contraceptive use has reduced maternal mortality. Matern Child Health J 2010;14:687-95.

4. Instituto Nacional de Estadísticas. Fecundidad en Chile. Enfoque estadístico. Boletín informativo del Instituto Nacional de Estadísticas. 7 de diciembre de 2006.

5. Donoso E, Becker J, Villarroel L. [Birth rates and reproductive risk in adolescents in Chile, 1990-1999]. Rev Panam Salud Publica 2003;14:3-8.

6. Donoso E, Villarroel L. [Reproductive risk of women over 40 years old]. Rev Med Chil 2003;131:55-9. 
7. Donoso E. [The reduction in maternal mortality in Chile, 1990-2000]. Rev Panam Salud Publica/Pan Am J Public Health 2004;15:326-30.

8. Chamy V, Cardemil F, Betancour P, Ríos M, Leigthon L. Riesgo obstétrico y perinatal en embarazadas mayores de 35 años. Rev Chil Obstet Ginecol
2009;74:331-8.

9. Ministerio de Educación, SERNAM. Educación sexual y afectividad. Hallado en: http://www.mineduc.cl/index2.php?id_contenido=13695\&id_portal=1\&id_seccion=10) 